Itinéraires Itinéraires

Littérature, textes, cultures

2011-1| 2011

Les Mémoires, une question de genre?

\title{
Redéfinir le holisme épique
}

\section{Marguerite Mouton}

\section{OpenEdition}

Journals

Édition électronique

URL : http://journals.openedition.org/itineraires/1649

DOI : $10.4000 /$ itineraires. 1649

ISSN : 2427-920X

Éditeur

Pléiade

Édition imprimée

Date de publication : 1 avril 2011

Pagination : 107-121

ISBN : 978-2-296-13692-2

ISSN : 2100-1340

Référence électronique

Marguerite Mouton, «Redéfinir le holisme épique », Itinéraires [En ligne], 2011-1 | 2011, mis en ligne le 01 avril 2011, consulté le 26 juin 2020. URL : http://journals.openedition.org/itineraires/1649 ; DOI : https://doi.org/10.4000/itineraires.1649

Itinéraires est mis à disposition selon les termes de la licence Creative Commons Attribution - Pas d'Utilisation Commerciale - Pas de Modification 4.0 International. 


\title{
Redéfinir le holisme épique
}

\begin{abstract}
At the end of the eighteenth century, philosophers started considering the history of literature as a linear progress towards a critical representation of society. Within this view, the epic genre was relegated to the past and thought to be out of date, even dead, since it was supposed to be the pure expression of the state of affairs in a period known as being holistic - when the mind was incapable of any critical approach of reality but was wholly engrossed in it. This article takes the idea that the epic is fundamentally linked to holism seriously, but offers a new interpretation of what this may mean and examines the consequences of such a definition for the history of the genre.
\end{abstract}

Keywords : epic, literary genres, history of literature, Hugo, Tolkien

Mots clés : épopée, genres littéraires, histoire littéraire, Hugo, Tolkien

Le terme épopée est fréquemment employé pour désigner de façon lâche des ouvres littéraires ou cinématographiques particulièrement spectaculaires et traversées par un certain souffle épique. De nombreux commentateurs, qu'ils soient journalistes ou universitaires, emploient en effet le terme epic, dont l'équivalent français oscille entre épopée et épique, pour qualifier des œuvres telles que Notre-Dame de Paris $^{1}$ de Victor Hugo, ou encore Le Seigneur des anneaux ${ }^{2}$ de J. R. R. Tolkien. L'usage du mot est très fréquent sous la plume des critiques; mais le terme n'a que rarement fait l'objet d'une étude spécifique. S'il s'agit bien souvent en français d'éviter une répétition malvenue, l'anglais (par exemple) ne partage pas le même souci et n'a donc pas cette excuse. Quelle que soit la langue, le choix de ce terme ne peut de toute façon

1. Victor Hugo, Notre-Dame de Paris, éd. Jacques Seebacher, Paris, Librairie générale française, 1999 - abrégé en NDP.

2. John Ronald Reuel Tolkien, The Lord of the Rings, Londres, Harper Collins, $2002-$ abrégé en LoR; Le Seigneur des anneaux, trad. Francis Ledoux, Paris, Christian Bourgois, 1992 - abrégé en $S d A$. 
être anodin, bien qu'il reste trop souvent impensé. À quelles conditions peut-on donc employer le terme d'épopée? Les théoriciens de l'épopée ont eu tendance à réduire le champ d'application du terme jusqu'à confisquer un genre généralement associé à une période révolue de l'humanité, caractérisée par ce qu'on appelle souvent le holisme.

\section{L'histoire mouvementée du genre}

Deux événements majeurs dans l'histoire de la théorisation de l'épopée ont contribué à la relégation du genre dans le passé et à son association avec la caractéristique holistique. Certaines philosophies de l'Histoire, qui apparaissent à partir des Lumières, ont ainsi imposé l'idée selon laquelle le genre de l'épopée serait lié à l'état du développement de l'esprit humain dans les périodes antique et médiévale et serait donc dépassé depuis la fin du Moyen Âge. Puis, dans les années 1970, la réflexion d'Étiemble sur l'extension du corpus épique a fixé des limites rigoureuses à l'emploi du terme épopée, qui ne désignerait plus que des œuvres appartenant à des époques anciennes, ou éventuellement à une littérature non-européenne. Ces deux étapes de la réflexion sur l'épopée contribuent à la confiscation de la catégorie générique, si bien que celle-ci est désormais quasiment interdite à la critique littéraire comme outil d'analyse d'œuvres postérieures à la période médiévale.

\section{Confiscation philosophique}

Au tournant du XIX ${ }^{e}$ siècle se développent, on le sait, des philosophies qui associent une pensée de l'évolution des genres littéraires à l'idée d'un progrès de l'humanité à travers l'Histoire ${ }^{3}$. Les théoriciens de cette période substituent ainsi à la classification traditionnelle l'idée d'une succession chronologique des genres. L'épopée est alors définie comme la forme $e^{4} \mathrm{de}$ la coïncidence parfaite de l'esprit humain aux conditions d'existence de son temps.

Dans la lignée de la pensée hégélienne, Lukács développe ainsi une analyse de ce qu'il évite d'appeler genre mais préfère nommer forme, dans la mesure où les œuvres littéraires ainsi désignées sont solidaires d'un certain stade de développement de l'esprit humain. Or la découverte de ces formes, c'est-à-dire la prise de conscience à la fois que le monde et les œuvres sont des éléments distincts, et qu'un lien existe entre ces œuvres épiques et un stade historique du monde, entraîne selon lui la mort de l'épopée ${ }^{5}$. En effet, à partir de cet événement, la production littéraire ne

3. Voir en particulier le Cours d'esthétique de Georg W. F. Hegel (trad. Jean-Pierre Lefebvre et Veronika von Schenck, Paris, Aubier, 1997 [1818-1829], vol. III).

4. Georg Lukács, Théorie du roman, trad. Jean Clairevoye, Paris, Denoël, 1968 [1920], p. 19.

5. Ibid., p. 25. 
sera plus une pure expression de la réalité, mais impliquera un recul critique par rapport à l'état des choses tel qu'il nous apparaît dans un premier temps. Ces découvertes philosophiques font entrer le monde dans une nouvelle période historique, celle qui correspond aux formes romanesques. Le genre du roman, qui, selon Lukács, est censé remplacer historiquement celui de l'épopée, serait la forme de la mise à distance critique de sa propre époque. Dorénavant, l'épopée apparaît donc comme un genre dépassé.

Cette pensée de l'histoire de l'esprit et des genres littéraires, qui traverse les Lumières et nourrit la réflexion du romantisme, signe jusqu'à nos jours l'acte de décès du genre épique aux yeux des théoriciens ${ }^{6}$. Et l'on a tendance à oublier les raisons philosophiques qui sont à l'origine d'une affirmation communément admise. Certes, il convient de prendre au sérieux la force de ce geste théorique qui a eu des effets durables sur la production littéraire; mais cette notion de mort de l'épopée est-elle à comprendre comme la clôture définitive de l'histoire du genre, ou comme un événement particulièrement important de celle-ci qui n'a pourtant pu suffire à éteindre toute aspiration épique chez les auteurs?

\section{Confiscation universitaire}

Le second acte de la confiscation de l'appellation d'épopée s'est joué au sein de la recherche universitaire, avec le « défi ${ }^{7}$ » lancé par René Étiemble en 1974. En déclarant à plusieurs reprises dans son article que, pour définir l'épopée, «il faut repartir de zéro ${ }^{8}$ », Étiemble a appelé à lutter contre l'ethnocentrisme européen par l'ouverture du corpus de référence à une multitude d'autres textes de la littérature mondiale. Un tel défi a rendu extrêmement difficile l'emploi rigoureux du terme d'épopée. Pour faire face à l'immensité de la matière, la critique a eu tendance à se pencher sur les textes les plus canoniques de chaque culture. En ce qui concerne l'Europe, les œuvres retenues sont peu nombreuses et souvent anciennes. En effet, pour parer à l'accusation de vouloir imposer une conception européenne comme universelle, les chercheurs préfèrent généralement s'en tenir aux œuvres reconnues comme épiques depuis toujours. Si l'esprit du défi d'Étiemble était donc de remettre en question l'universalité d'un modèle, la recherche d'une définition valable pour toute la littérature mondiale

6. Cette conception trouve également des échos chez des penseurs plus récents tels que Michel Foucault pour qui, on le sait, l'avènement de la Renaissance correspond au divorce des mots et des choses, à la fin de l'immédiateté du rapport entre le monde et les écrits que celui-ci voit naître (Les Mots et les choses. Une archéologie des sciences humaines, Paris, France loisirs, 1990 [1966]).

7. Florence Goyet, «L'épopée », Vox Poetica (http://www.vox-poetica.com/sflgc/biblio/ goyet.html), 25 juin 2009, consulté le 19 mai 2010.

8. René Étiemble, «L'épopée de l'épopée », dans Essais de littérature (vraiment) générale, Paris, Gallimard, 1974. 
a peut-être au contraire conduit à une autre forme d'absolutisme : elle a interdit de parler d'épopée à cause de l'ampleur et de la variété du corpus, ou a réservé le terme à l'étude de textes lointains dans le temps ou dans l'espace, au détriment d'œuvres plus proches.

Entre condamnation et confiscation, l'épopée n'est pas pour autant

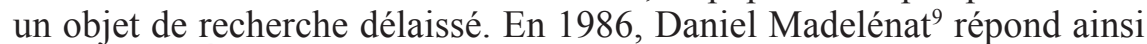
à l'appel d'Étiemble en proposant un panorama de l'épopée à l'échelle mondiale; Jean-Marcel Paquette a pris sa suite en $1988^{10}$ en élargissant son étude de l'épopée médiévale à une réflexion plus globale; plus récemment, un programme de recherche actif s'est mis en place autour de Dominique Boutet ${ }^{11}$, et des thèses sont régulièrement soutenues ${ }^{12}$. Ces travaux présentent néanmoins deux caractéristiques héritées de la théorie de la mort de l'épopée ou des répercussions du défi d'Étiemble. La plupart d'entre eux se concentrent en effet sur l'extension spatiale d'un corpus qui reste généralement composé d'œuvres antérieures à l'époque moderne et demeure ainsi tributaire d'une conception où l'épopée est liée à un état ancien de la pensée et de la société.

\section{Un genre holistique?}

Il convient néanmoins de mentionner la thèse de Florence Goyet et la façon dont son analyse du genre épique remet en cause l'idée hégélienne selon laquelle les hommes de l'Antiquité ou du Moyen Âge auraient été dépourvus d'esprit critique ${ }^{13}$. Relevant le défi d'Étiemble avec un corpus ancien et aux dimensions mondiales, F. Goyet propose la notion de travail épique, qui met à mal l'idée convenue que l'épopée est la forme de l'adéquation de l'esprit humain à l'état du monde d'une époque. Au contraire, le propre du genre serait de s'inscrire en faux par rapport à son temps en offrant un modèle idéologique alternatif. L'épopée, même ancienne, ne

9. Daniel Madelénat, L'Épopée, Paris, PUF, 1986.

10. Jean-Marcel Paquette, Typologie des sources du Moyen Âge occidental, Turnhout, Brepols, 1988.

11. Dominique Boutet anime ainsi le GREp, Groupe de Recherche sur l'Épique fondé en 1991 par François Suard. Il a publié un certain nombre d'ouvrages sur l'épopée, dont les actes d'un colloque organisé avec Camille Esmein-Sarrasin, intitulé Palimpsestes épiques. Récritures et interférences génériques (Paris, Presses de l’Université Paris-Sobonne, 2006). 12. Dans un autre domaine, la question des avatars poétiques de l'épopée dans la littérature moderne a été soulevée en 2008 par Delphine Rumeau dans sa thèse de doctorat (Chants du Nouveau Monde. Épopée et modernité, Whitman, Neruda, Glissant, Paris, Classiques Garnier, 2009). Le programme de Littérature Générale et Comparée des sessions 2010 et 2011 de l'Agrégation de Lettres Modernes remet à l'honneur le genre en s'interrogeant sur la « permanence de la poésie épique au Xxe siècle » (à partir d'œuvres d'Anna Akhmatova, Nâzim Hikmet, Pablo Neruda et Aimé Césaire).

13. Florence Goyet, Penser sans concepts : fonction de l'épopée guerrière, Paris, Champion, 2006. 
serait donc pas holistique au sens où elle serait immergée dans la société de son temps. Dès lors, deux conceptions de l'épopée s'affrontent : l'une défend un holisme de l'épopée, l'autre attribue au genre la capacité de dépasser une pure expression directe du monde.

Par rapport à ce débat sur la définition du genre, plusieurs positions sont possibles concernant la théorie de la mort de l'épopée. Cédric Chauvin part ainsi du principe qu'il n'y a plus d'épopées ${ }^{14}$. À partir d'un corpus issu de la littérature de la seconde moitié du $\mathrm{XX}^{\mathrm{e}}$ siècle, il pense les résurgences de l'épique à l'époque contemporaine à travers la notion de fonction de la référence épique. Cet outil lui permet de rendre compte de la façon dont chaque auteur tire profit, à l'intérieur de son roman, des motifs traditionnels d'un genre dont la disparition permet une certaine liberté de maniement rétrospectif. La référence épique est donc désormais circonscrite dans des motifs traditionnels insérés à l'intérieur d'œuvres romanesques.

Une autre option consiste à prendre au sérieux à la fois l'idée répandue que le holisme est constitutif du genre et la thèse selon laquelle l'épopée n'est pas l'expression immédiate de l'état d'une société à une époque donnée. C'est à une réinterprétation du holisme non plus comme immersion de l'esprit dans son temps mais comme tendance hégémonique interne au genre que je voudrais procéder ici. Il me semble en effet que l'on peut décliner trois modèles de compréhension du holisme épique. Tout d'abord, l'histoire que l'épopée raconte ne se déroule pas dans le temps, mais dans un cadre mythique, au sens où, sous une coloration passée, l'époque de l'action est définie par la façon dont elle correspond parfaitement à un état de l'esprit, loin des contingences d'une temporalité historique. L'épopée est en effet immergée dans une unité sémantique et exclut ainsi l'ironie et toute mise à distance comique de l'univocité mimétique qui caractérise sa façon de représenter le monde. Enfin, l'épopée rend compte de la totalité de l'état du monde et ne supporte pas la fragmentation. Pour conclure à la mort de l'épopée, il faudrait donc pouvoir montrer qu'il n'existe pas d'œuvre moderne qui ne contrevienne à chacune des trois dimensions de ce que l'on peut appeler le holisme constitutif de l'épopée. Dans le cas contraire, il faudrait en effet conclure à un certain degré de persistance de ce qui constitue en propre l'épopée, et donc peut-être à une forme de permanence du genre.

\section{Les limites du holisme dans l'« épopée moderne »}

Pour déterminer si l'épopée est morte ou bien s'il est possible d'y rattacher des textes moins canoniques, plus modernes et appartenant à la culture européenne, j'étudierai la façon dont ces différentes définitions du

14. Cédric Chauvin, Statuts et fonctions de la référence épique en France depuis la Seconde Guerre mondiale, Paris, Champion, 2010. 
holisme fonctionnent à l'intérieur d'un corpus composé de Notre-Dame de Paris de Victor Hugo et du Seigneur des anneaux de J. R. R. Tolkien. Ces œuvres, écrites en France et en Angleterre aux $\mathrm{XIX}^{\mathrm{e}}$ et $\mathrm{XX}^{\mathrm{e}}$ siècles, reprennent en effet un certain nombre de motifs traditionnels de l'épopée et en particulier de la chanson de geste médiévale, et elles sont fréquemment désignées par la critique comme « épopée », sans que cette appellation fasse l'objet d'une théorisation.

\section{Le modèle de l'immersion mythique}

L'enquête sur le premier aspect du holisme épique ne plaide pas en faveur de la thèse d'une survie de l'épopée. En effet, les œuvres postérieures à la mort de l'épopée que sont Notre-Dame de Paris et Le Seigneur des anneaux ne sont pas totalement immergées dans un passé glorieux, mais mettent en perspective l'ancien temps à l'intérieur même de la fiction en introduisant la dimension du présent. Certes, le conteur de l'épopée traditionnelle établissait un lien entre l'histoire passée et son récit présent ; mais la diégèse même des textes de Hugo et de Tolkien met en scène une sortie du passé pour rejoindre le présent. Les deux œuvres réfléchissent sur le temps; or c'est cette distance réflexive qui marque la disparition de l'épopée selon la tradition hégélienne. En effet, chez Tolkien comme chez Hugo, le passé est représenté en devenir : leurs deux œuvres ont pour cadre une période de grand changement.

Notre-Dame de Paris a pour thème le bouleversement politique et développe une double pensée de l'Histoire. La première rappelle que la fin du monde féodal marque le début de la monarchie absolue et du pouvoir centralisé. Le choix de l'année 1482 comme sous-titre de Notre-Dame de Paris est significatif : située un an avant la mort de Louis XI, cette date marque le basculement entre la juridiction féodale et un nouvel ordre politique, celui de la monarchie absolue, qui concentre les pouvoirs en une seule personne. Le nouveau régime est en effet préfiguré dans cette réplique du roi à qui l'on vient de rapporter le soulèvement populaire contre la cathédrale : « il faudra bien que le jour vienne où il n'y aura en France qu'un roi, qu'un seigneur, qu'un juge [...], comme il n'y a au paradis qu'un Dieu ${ }^{15}$ ! » La comparaison renvoie directement à la conception d'une monarchie absolue de droit divin. La représentation hugolienne de l'Histoire ouvre également une perspective à plus long terme et fait succéder la démocratie à la théocratie, sur le modèle de la révolution artistique qui substitue la liberté à la contrainte architecturale : à l' " unité » du dogme théocratique qu'incarne le lourd plein-cintre de la cathédrale romane s'oppose « la fantaisie et le caprice " du peuple, que représente l'ogive légère de l'architecture gothique $^{16}$. Le passé n'est plus représenté pour lui-même, mais dans un 
échange avec le présent (celui de la période postérieure au Moyen Âge) qu'il a pour fonction d'éclairer.

La diégèse du Seigneur des anneaux fait quant à elle basculer la Terre du Milieu dans une nouvelle ère, le quatrième âge; or celui-ci sera dominé par les Hommes et non plus par des peuples imaginaires tels que les Elfes ou les Ents ${ }^{17}$. En effet, les personnages et situations qui demeurent dans la dernière scène du roman sont très proches du présent du lecteur ${ }^{18}$. Sam retrouve sa famille et la perspective d'une vie ordinaire dans un village de la Comté, pays le moins étrange de la Terre du Milieu. Tolkien soutient en effet que " "la Comté" a pour modèle l'Angleterre rurale et aucun autre pays au monde ${ }^{19} »$. Le lien que l'auteur établit dans sa correspondance entre la réalité la plus ordinaire de sa propre vie et l'univers fictionnel éloigne le présent des Hobbits du cadre mythique. La fin du troisième âge inscrit donc dans la fiction à la fois la sortie d'un passé associé aux caractéristiques traditionnelles de l'épopée (le combat héroïque), et l'entrée dans le présent. L'introduction d'une évolution temporelle dans les textes modernes met donc en péril l'immersion holistique dans un passé mythique.

\section{Le modèle de l'unité sémantique}

En instaurant une distance avec le passé, les œuvres de Hugo et de Tolkien s'écartent également d'une deuxième conséquence logique de la définition de l'épopée comme la forme adéquate à une époque. À la mise en perspective temporelle s'ajoute en effet une mise en question de l'unité sémantique du monde.

Dans une analyse désormais classique, Verlyn Flieger montre ainsi comment la théorie linguistique de "l'ancienne unité sémantique » développée par Owen Barfield ${ }^{20}$ fournit un cadre à cette pensée de la perte de l'immédiateté originelle, par le biais de l'introduction d'une dimension temporelle dans l'œuvre et des contingences qui l'accompagnent ${ }^{21}$. Le point de départ de la théorie de Barfield telle que la rapporte Flieger est une

17. SdA, p. 1044 ; LoR, p. 957.

18. Voir Vincent Ferré, Tolkien : sur les rivages de la Terre du Milieu, Paris, Christian Bourgois, 2001, p. 23.

19. " "The Shire" is based on rural England and not any other country in the world (The Letters of J. R. R. Tolkien, Humphrey Carpenter (éd.), Londres, Harper Collins, 2006, p. 249; édition française : J. R. R. Tolkien, Lettres, trad. Delphine Martin et Vincent Ferré, Paris, Christian Bourgois, 2005 - abrégé en Letters. Les numéros de pages renvoient à l'édition anglaise).

20. Ami de Tolkien et membre du groupe des Inklings (qui se réunissait pour discuter des œuvres de chacun des membres), Owen Barfield est l'auteur de Poetic Diction : a Study in Meaning (Londres, Faber \& Gwyer, 1928).

21. Verlyn Flieger, Splintered Light : Logos and Language in Tolkien's World, Kent, Kent State University Press, 2002 [1983]. 
description de la relation de l'homme à son environnement, qui implique à la fois la conscience qu'il en a et la façon dont celle-ci s'exprime dans des mots ${ }^{22}$. Dans le cas du développement de la conscience comme dans celui du langage, s'opposent d'une part un état originaire unifié et, d'autre part, un état postérieur éclaté. Au départ, l'humanité percevait le cosmos comme un tout et elle-même comme une partie du tout. Par concomitance, toute parole était littérale et était l'expression directe de la perception d'un phénomène. Le langage ne faisait pas de distinction entre les sens littéral et métaphorique. À présent, nous percevons le cosmos à travers des éléments particuliers, fragmentaires et séparés de nous. Du point de vue du langage, la distinction moderne entre le sens littéral et le sens métaphorique suppose une séparation par rapport à l'objet concret, une capacité d'abstraction. Notre conscience et le langage dans lequel nous l'exprimons ont changé ensemble dans le sens d'un éclatement, mais aussi d'un affaiblissement des mots : ceux-ci ne permettent plus de saisir l'unité sémantique forte des origines.

La thèse de Flieger montre comment la théorie de Barfield informe la fiction de Tolkien. La technique de celui-ci consiste à donner une littéralité, dans ce qu'il appelle le Monde Secondaire ou fictionnel, à ce qui serait appelé métaphore dans le Monde Primaire ou réel; il illustre ensuite le procédé par lequel le littéral devient métaphorique. Ainsi, de manière symbolique, plus les peuples du Monde Secondaire s'éloignent géographiquement de la source de lumière originelle au cours de leur histoire, plus ils se subdivisent en races différentes, parlant des langages distincts et possédant des attributs et aptitudes variés. Et ces peuples, langues et qualités perdent en complexité et en noblesse avec l'éloignement. Selon cette interprétation, Tolkien raconterait donc l'histoire d'un éclatement de l'humanité en une multitude de peuples. Le résultat final de ces principes de dispersion et d'affaiblissement réside alors dans les plus petits fragments, que sont les Hobbits. Expression d'une distinction dans le concept d'humain, ils incarnent parfaitement l'humanité ordinaire, ce que manifeste symboliquement leur habitude de marcher pieds-nus.

Or le petit peuple des Hobbits, résultat ultime de la séparation d'avec l'unité originelle, brise le sérieux de l'épopée traditionnelle et introduit dans l'œuvre une dimension comique, propre à la conception moderne du héros ${ }^{23}$. L' « anachronisme» que Bilbo constitue, selon Tom Shippey, éminent spécialiste de l'œuvre de Tolkien, et que les Hobbits du Seigneur des anneaux perpétuent, fait voler en éclats l'unité de sens de l'épopée, en « exprim[ant] l'opinion moderne, les incapacités modernes ». Cette tendance à la particularisation comique de Hobbits qui fument la pipe au milieu des « épaves ${ }^{24}$ » d'un champ de bataille s'oppose à l'unité d'un sens

22. Verlyn Flieger, op. cit., p. 33-44.

23. Tom Shippey, The Road to Middle-earth, Londres, Harper Collins, 1992 [1982], p. 71.

24. $S d A$, livre III, chap. IX. 
noble. Le Seigneur des anneaux contrevient donc à la deuxième caractéristique du holisme épique traditionnel, en remettant en cause l'unité sémantique du monde représenté.

Victor Hugo va encore plus loin dans le développement de la veine comique, alors même que la préface de Cromwell associe la disparition de l'unité originelle du monde grec et le grotesque qui informe la littérature moderne. On a souvent souligné la façon dont Notre-Dame de Paris s'inscrivait tout particulièrement dans la veine burlesque et son corollaire héroïcomique, en particulier en ce qui concerne les personnages de Phœbus, de Gringoire et de Jehan Frollo. Les trois hommes constituent une mise à distance du héros aristocratique classique et déclinent sous des formes variées ce renversement du protagoniste masculin de l'épopée traditionnelle. Jehan Frollo fait ainsi l'objet d'une véritable déconstruction du motif épique. Le topos de l'équipement du guerrier devient l'occasion de rire du manque de virilité du personnage, de sa « voix en flûte qui s'échappait de dessous une pesante armure ${ }^{25} \gg$ et de son visage qui renverse le blason médiéval héroïque et l'échange contre la description canonique de l'enfant. Le burlesque passe par les connotations négatives et le registre familier de la description de l'armure, "panoplie " "vissé[e] sur le corps », « pesante » et « rouillée », avec une ceinture « pleine de » dagues et d'épées. L'énumération des composantes de l'équipement se termine par la mention: « et un vaste broc de vin devant lui, sans compter à sa droite une épaisse fille débraillée ». Ces derniers attributs sont mis sur le même plan que les autres éléments par la récurrence de compléments de lieux (《sur le flanc», « à sa gauche », puis « devant lui » et « à sa droite») qui organisent la description. De même, par un renversement parodique, la chanson qu'entonne Jehan face à la mort, loin d'être l'expression de la furie héroïque, n'est qu'une simple comptine accompagnée d'un « rire effronté » et chantée « avec son intrépide insouciance d'enfant de seize ans ${ }^{26} »$. Le narrateur note qu'il s'agit d'une « chanson alors populaire ». Le complément de temps renvoie ironiquement au Moyen Âge, alors même que la chanson ne correspond pas à la tonalité commandée par la scène dans un contexte médiéval. Le rire fait éclater l'unité sémantique et l'immédiateté sur lesquelles repose traditionnellement le fonctionnement épique, menaçant par là la persistance du genre.

L'épopée est donc minée par deux phénomènes : celui de la mise en perspective qui crée une distance critique et celui de la fragmentation du sens. Faut-il pour autant en conclure à une mort de l'épopée, quitte à admettre la possibilité de manifestations insulaires de l'épique à l'intérieur d'œuvres modernes? C'est dans cette voie que s'engage Cédric Chauvin, en 
développant les notions de registre et de fonction de la référence épique ${ }^{27}$. Prenant au sérieux l'affirmation théorique selon laquelle l'épopée est morte, il est ainsi conduit à limiter le champ d'application de la tradition épique. Dans ce contexte, le genre perd alors toute dimension holistique et l'utilisation qui en est faite s'inscrit en rupture par rapport à la définition traditionnelle du genre. Cependant, ne peut-on au contraire maintenir un troisième modèle de holisme qui résiderait dans l'ambition compréhensive d'un genre excluant la simple réalisation fragmentaire à l'intérieur du roman?

\section{La tendance hégémonique du genre épique?}

L'étude de Notre-Dame de Paris et du Seigneur des anneaux fait apparaître une autre définition du holisme, caractérisée par une tendance hégémonique. En effet, loin de se réduire à des réalisations fragmentaires, l'épopée a pour enjeu la représentation complète d'un monde et est, dans ce cadre, capable d'accueillir sous son égide unificatrice d'autres genres littéraires.

\section{Victor Hugo : la définition d'un genre nouveau}

Le problème du rapport entre unité et diversité génériques est au cœur des débats soulevés par la critique autour de Notre-Dame de Paris. De nombreux commentateurs de Victor Hugo, et parmi eux William Holdheim, reprochent à l'œuvre un manque d'unité ${ }^{28}$, qui tiendrait à l'intrication de plusieurs genres littéraires, et en particulier à l'ajout de passages apparemment sans rapport avec l'intrigue dans l'édition de 1832. Au contraire, lorsque Hugo ajouta ces chapitres, il prit grand soin d'affirmer que ceux-ci étaient issus « de la même pensée ${ }^{29}$ » que le reste du livre. Cette revendication dépasse la dimension purement chronologique pour inviter le lecteur à rechercher « la pensée d'esthétique et de philosophie cachée dans ce livre $^{30} »$.

Holdheim n'est cependant pas insensible à cet appel à une interprétation globale et unifiée, quoique non manifeste, de l'œuvre. Il rejoint ainsi paradoxalement une autre tendance de la critique, qui a cherché à lire Notre-Dame de Paris comme une épopée, entendue comme une œuvre d'art

27. Cédric Chauvin, op. cit., p. 67.

28. Voir William Holdheim, «The History of Art in Victor Hugo's Notre-Dame de Paris » [1976-1977], dans William Holdheim (ed.), The Hermeneutic Mode. Essays on Time in Literature and Literary Theory, Ithaca, Cornell University Press, 1984, p. 94. Cette idée constitue le point de départ de beaucoup d'études, par exemple celle de Kathryn Wildgen dans « Romance and Myth in Notre-Dame de Paris » (French Review, n 49, février 1976, p. 319-327).

29. NDP, p. 55.

30. NDP, p. 56-57. 
complète, synthèse unifiant tous les genres. En effet, le «but de l'auteur » que la note ajoutée à l'édition de 1832 invite le lecteur à rechercher est, selon lui, de produire une «nouvelle épopée ${ }^{31} »$. Holdheim n'en conclut pas moins à l'échec de Notre-Dame de Paris, qui n'a pas réussi à remplir ce programme et n'a pas su donner vie à la cathédrale. Le critique pose néanmoins en 1976 les fondements d'une interprétation à plus haut sens de la question générique dans Notre-Dame de Paris autour de la notion d'épopée. Une telle lecture sera reprise et menée vers une conclusion positive par des critiques tels que Illinca Zarifopol-Johnston en 1985 et Brigitte Jacobsen en $1993^{32}$. Ces commentateurs s'appuient sur la définition de la note de Victor Hugo « Sur Walter Scott » :

\footnotetext{
Après le roman pittoresque, mais prosaïque, de Walter Scott, il restera un autre roman à créer, plus beau et plus complet selon nous. C'est le roman à la fois drame et épopée, pittoresque mais poétique, réel mais idéal, vrai mais grand, qui enchâssera Walter Scott dans Homère ${ }^{33}$.
}

Paradoxalement, Holdheim et ses continuateurs ont pu voir dans cette citation la définition d'une épopée, et non d'un roman ${ }^{34}$. Il faut croire qu'ils ont considéré que le "roman » que Hugo mentionne ici a plus à voir avec le genre de l'épopée qu'avec celui du roman : il s'agirait en effet d'une désignation vide dont le contenu est fourni par la fin de la phrase qui l'assimile à une réalisation particulière de l'œuvre d'Homère. Selon cette lecture, l'emploi du terme « roman » de préférence à celui d'épopée peut tout au plus renvoyer au fait que l'épopée ainsi définie se distingue de l'épopée traditionnelle, dont elle est l'avatar moderne. Si l'on accepte de prendre au sérieux le fait que le «roman» dont il est question est une épopée, il faut alors convenir que Hugo propose une définition paradoxale de ce genre total : le terme épopée (que l'on peut substituer dans le texte à celui de roman) recouvre "à la fois » l'épopée elle-même et le drame. Or comment un élément peut-il, en toute rigueur, être à la fois lui-même et autre chose? La contradiction est levée si l'on considère que dans l'équation simplifiée Épopée $=$ Épopée + Drame, la deuxième occurrence du mot épopée désigne le genre représenté par l'œuvre d'Homère, et la première un genre typiquement hugolien. La question est alors de savoir comment Hugo définit et réalise cette épopée nouvelle.

Une clé en est donnée dans l'analogie entre l'épopée définie par la préface de Cromwell et la cathédrale telle qu'elle est figurée dans Notre-

31. William Holdheim, op. cit., p. 108.

32. Brigitte Jacobsen, « Reconstruction et vision de l'histoire dans Notre-Dame de Paris », dans Gérard Laudin et Edgar Mass (dir.), Représentation de l'histoire. Actes du colloque franco-allemand de Cologne des 17-18 juin 1988, Cologne, Janus, 1993, p. 147-157.

33. Victor Hugo, Euvres complètes, vol. « Critique », Paris, Robert Laffont, 1985, p. 149. 34. Au contraire, Henri Scepi voit dans cette citation la définition non ambiguë du roman (Notre-Dame de Paris de Victor Hugo, Paris, Gallimard, coll. « Foliothèque », 2006, p. 41). 
Dame de Paris. Hugo décrit de la même façon l'opposition entre les styles architecturaux romans et gothiques qui composent la cathédrale et leurs équivalents littéraires, épopée et drame, décrits dans la préface de Cromwell. I. Zarifopol-Johnston propose de considérer cette analogie en termes de structures identiques ${ }^{35}$. Le style roman est en effet dépeint comme une structure répétitive et immobile, liée à la fatalité, analogue à celle de l'épopée, succession d'épisodes sans progression linéaire dont la seule fin est la mort. Le gothique, à l'instar du drame, est quant à lui le style de la liberté, de l'émotion, de la légèreté et de la hauteur. Du point de vue de la structure, le drame présente des scènes, des événements et non plus une succession sans progression. L'épopée hugolienne, sur le modèle de la cathédrale de Paris, serait alors précisément le genre qui intègre cette opposition des genres et des styles.

Le lien entre épopées homérique et hugolienne est plus qu'une homonymie. Le choix de désigner le genre englobant comme épopée met en avant une caractéristique du genre épique. Celui-ci vise en effet une forme de holisme, d'enveloppement de l'ensemble des possibles. Cette théorisation générique fournit des outils pour mieux comprendre la configuration d'autres œuvres épiques modernes, telles que celle de Tolkien.

\section{J. R. R. Tolkien : le contraste générique}

À l'éditeur de l'Observer, Tolkien écrit en 1938 que son œuvre est issue « de l'épopée, de la mythologie et du conte de fées (préalablement digérés $)^{36} \gg$. On peut ajouter à cette liste le romance ${ }^{37}$, et la critique a cru identifier d'autres caractéristiques génériques de son œuvre dans la littérature moderne, telles celles du roman gothique, ou de la poésie moderniste ${ }^{38}$. Contrairement aux apparences, la conférence «Du Conte de fées » ne permet pas de trancher en faveur du conte de fées, mais se contente d'emprunter à G. Dasent l'image d'une « soupe » dont on ne cherche pas à distinguer les composantes, mais que l'on apprécie de manière globale ${ }^{39}$. Faut-il donc renoncer à identifier l'épopée? L'image prosaïque de la soupe ne doit pas nous tromper : elle relève d'une posture de Tolkien qui entre

35. Illinca Zarifopol-Johnston, « Notre-Dame de Paris. The Cathedral in the Book », Nineteenth Century French Studies, n 13/2-3, 1985, p. 28.

36. « [F]rom (previously digested) epic, mythology, and fairy-story » (Letters, p. 31).

37. Letters, p. 414.

38. Martin Simonson, The Lord of the Rings and the Western Narrative Tradition, Zurich, Iéna, Walking Tree Publishers, 2008, p. 56-59. Voir aussi Lin Carter, Tolkien, A Look behind The Lord of the Rings, New York, Ballantine Books, 1969; édition française : Tolkien, le maître des anneaux, trad. Dominique Haas, Saint-Amand-Montrond, Le Pré aux Clercs, 2003, p. 137-166.

39. J. R. R. Tolkien, On Fairy-stories, éd. Verlyn Flieger et Douglas A. Anderson, Londres, Harper Collins, 2008, p. 39, § 25. 
dans la construction globale d'un mythe de l'écriture dont ni auteur ni critique ne pourrait percer le mystère, d'un texte qui « coule maintenant tout seul et échappe totalement [au] contrôle ${ }^{40} \gg$. De plus, le motif même de la soupe fournit un moyen de penser la force d'une unité globale de l'œuvre susceptible d'accueillir des genres multiples et confus, mais dont l'intérêt pour le lecteur réside dans l'ensemble qui en résulte. Or c'est précisément la définition de l'épopée telle qu'elle est apparue à travers l'étude de NotreDame de Paris.

À partir de ces considérations, il est possible de renverser l'analyse menée par V. Flieger dans son fameux article «Aragorn et Frodo : le concept du héros ${ }^{41} »$, en prenant pour point de départ non pas le début de sa réflexion, mais la fin, qui autorise une autre lecture de son commentaire. En ouverture, Flieger affirme qu'elle n'a « pas l'intention de ranger Le Seigneur des anneaux dans un genre particulier ${ }^{42} \gg$. La dernière phrase de l'article ouvre la voie à un autre modèle de fonctionnement générique que celui de "n'appartenir qu'à une de ces catégories »: «En nous donnant à la fois Aragorn et Frodo, [Tolkien] a exploité le contraste qu'ils présentent pour élargir et approfondir la signification de leur histoire ${ }^{43}$. » Flieger reprend la terminologie proposée par Northrop Frye ${ }^{44}$. Le personnage d'Aragorn correspondrait ainsi au mode mimétique supérieur, c'est-àdire au héros d'épopée ou de romance, qui suscite l'admiration du lecteur, sans permettre l'identification. Frodo serait à rapprocher au contraire du mode mimétique inférieur que l'on trouve par exemple dans le conte de fées. Personnage ordinaire, il favorise l'identification, bien qu'il soit projeté dans une action qui relève du mode mimétique supérieur. Ici perce déjà une complexification du modèle.

Flieger procède ensuite à une distinction entre caractérisation « profonde ${ }^{45}$ » et « superficielle ${ }^{46}$ » des deux héros. À l'évidence, il s'agit de penser une coexistence générique à l'intérieur de chaque personnage. Aragorn est un héros d'épopée, mais aussi de romance; Frodo appartient au conte de fées, mais "incarne des éléments mythiques et héroïques 》 et est finalement «associ[é] au héros épique médiéval ${ }^{47}$ ». Cet apparent

40. « It is now flowing along, and getting quite out of hand (Letters, p. 40).

41. Verlyn Flieger, « Frodo and Aragorn : the Concept of the Hero », dans Neil D. Isaacs et Rose A. Zimbardo (ed.), Tolkien : New Critical Perspectives, Lexington, University Press of Kentucky, 1981, p. 40-62; « Frodo et Aragorn : le concept du héros », trad. Pascal Aubin, dans Vincent Ferré (dir.), Tolkien, trente ans après, Paris, Christian Bourgois, 2004, p. 253-278.

42. Verlyn Flieger, « Frodo et Aragorn... », op. cit., p. 253.

43. Ibid., p. 278.

44. Northrop Frye, Anatomy of Criticism. Four Essays, Princeton, Princeton University Press, 1990 [1957], p. 56-62.

45. Verlyn Flieger, «Frodo et Aragorn... », op. cit., p. 264.

46. Ibid., p. 266.

47. Ibid., p. 277. 
parallélisme inversé des structures décrites par Flieger masque en fait un déséquilibre en faveur de l'épopée, qui intervient dans la caractérisation des deux personnages, tandis que l'autre genre avec lequel il se combine est, suivant le cas, le romance ou le conte de fées. Par ailleurs, le lien qui unit romance et épopée dans le cas d'Aragorn est un lien de « subordination $^{48} \gg$ du premier genre au second. La réciproque n'est pas vraie pour Frodo : l'épopée n'est pas inféodée au conte de fées; mais Flieger a du mal à qualifier clairement le lien qui unit les deux genres. Elle hésite à y voir une relation de succession entre le conte de fées et l'épopée, un processus d' « agrandissement » de Frodo; pourtant, affirme-t-elle, le personnage « conserve sa cohérence avec ce qu'il a été dès le début ${ }^{49}$ ". Il semble donc nécessaire de penser un lien de coprésence des genres. Or V. Flieger parle d'une synthèse entre des éléments structuraux, d'une part, et des traits psychologiques qui caractérisent le héros de conte de fées, d'autre part. Il semble donc que la coexistence des deux genres soit permise par la distinction de leur domaine d'application.

Flieger est ici gênée par le parti-pris de travailler sur la caractérisation générique des héros et non sur le récit de leurs aventures. Le personnage de Frodo possède les caractéristiques propres au héros de conte de fées, mais est placé dans un récit épique. Cette description comporte de nouveau un déséquilibre en faveur de l'épopée, qui est du côté de la «structure » intégrant l'altérité générique du personnage. Cependant, ces déséquilibres font apparaître une autre définition : loin d'être un genre parmi d'autres, l'épopée est le seul capable d'accueillir le conte de fées ou le romance, dans la mesure où il est la forme du contraste entre les éléments. La force même de la disjonction entre la tradition du héros épique appelée par le genre des aventures du personnage et la caractérisation de celui-ci comme héros de conte de fées produit le « contraste » propre à l'épopée moderne. C'est justement parce que l'aventure de Frodo « est terriblement inappropriée $^{50} \gg$ au personnage, du fait même du décalage entre les genres, que le récit accède au statut d'épopée.

Le caractère d'épopée du Seigneur des anneaux apparaît donc non seulement à un premier degré dans la prééminence du genre dans le personnage d'Aragorn, mais plus particulièrement à un second niveau dans le contraste entre Aragorn et Frodo, voire finalement dans la contradiction entre le profil narratif de ce dernier et les événements auxquels il est confronté. En contrepartie, l'épopée se trouve redéfinie par le contact avec les genres qu'elle intègre : d'une part, elle prend le statut de genre accueillant aux autres genres, d'autre part elle acquiert, à travers le filtre de l'antithèse qui la constitue, les qualités propres aux genres qu'elle inclut.

48. Verlyn Flieger, « Frodo et Aragorn... », op. cit., p. 263.

49. Ibid., p. 266.

50. Ibid., p. 277. 
Cette étude a cherché à examiner la pertinence de l'emploi du terme épopée pour qualifier des œuvres modernes telles que Notre-Dame de Paris et Le Seigneur des anneaux, en analysant la façon dont celles-ci répondent aux attendus littéraires, mais aussi philosophiques du genre. L'épopée a en effet été théorisée par les penseurs des Lumières qui considèrent l'histoire de la littérature comme le déploiement linéaire du progrès de l'esprit humain. Ce dernier s'arracherait en effet à sa condition d'immersion dans la société de son temps pour acquérir un regard critique sur le réel et finalement conférer une autonomie au texte, qui ne serait plus pure expression du monde comme l'était l'épopée. Il semble bien que cette immédiateté soit en effet définitivement écartée. Cependant, une analyse plus approfondie offre le moyen de penser la persistance d'un type d'œuvres qui conserve l'ambition compréhensive de dire un monde complet quoique celui-ci soit en décalage par rapport au réel - à condition de pouvoir concevoir une œuvre totalement épique, et non une fonction circonscrite du genre à l'intérieur d'une œuvre qui lui est hétérogène.

Marguerite Mouton

Université Paris 13 